\title{
Cost Effective Production of Penicillin and Biogas from Rotten Oranges by Microbial Fermentation Technique
}

\author{
Aradhana Priyadarsini ${ }^{1}$, A. T. Vivek ${ }^{2}$, C. Gopinathan ${ }^{3}$ \\ 1,2, ${ }^{3}$ Bioprocess laboratory, Department of Biotechnology, University of Calicut, Tenjhipalam, P.O. -673635,Kerala, India
}

\begin{abstract}
India produces 10.8 million tonnes oranges annually, of which approximately $30 \%$ goes as waste. Orange juice primarily contains sucrose, fructose and glucose as carbon sources which can be fermented to Penicillin and biogas. During the processing of orange juice, huge quantities of orange peel and pulp are generated which causes environmental pollution problem. This can be converted to biofuel (Biogas) by anaerobic technology (methanogenesis). Different media formulations were prepared with peptone and ammonium sulphate by performing agar well diffusion assay of the respective fermentation broths by preparing cell free extracts and the potency of each of the formulations was tested. The diameter of inhibition zone was taken as a measure of potency of each of the formulation using raw orange juice as the basic substrate. It was found that compared to conventional media using raw orange of pH 3.5 , orange juice adjusted to $\mathrm{pH} 6.5$, peptone added and ammonium sulphate added formulations gave an inhibition zone of diameters, $40 \mathrm{~mm}, 45 \mathrm{~mm}$ and $41 \mathrm{~mm}$ respectively.The biogas production from orange peel was found to be $68.9 \%$ more compared to control.
\end{abstract}

Keywords: Penicillin, Rotten/Waste oranges, mat culture, Agar-well diffusion assay, Biogas, Anaerobic digestion.

\section{Introduction}

The production of orange juice on a large scale leads to significant level of solid and liquid remains (20 million tonnes/year), which is accounted as waste. Since their composition is rich in pectin, cellulose and hemicellulose, and essential oils; these could be utilized for several industrial processes since these orange remains have no economic value. Orange is a citrus fruit, grown in high quantities globally, mostly exists in terms of peeled oranges and as juice [1]. It is linked to low cost and it consists of vital nutrients including dietary fiber, Vitamin A, B, C, minerals (calcium, phosphorus, potassium), and various phytochemicals such flavanoids, carotenoids and amino acids [2]-[3].

Brazil is the largest producer of orange with $53 \%$ orange juice globally and $80 \%$ of international trade of orange related products [4]. It is estimated that half the fresh orange weight is transformed into juice during orange juice production and remaining weight is assumed to be waste [5]. Hence, this huge amount of waste can be converted to many value added products using applicable fermentation techniques. Since orange juice contains sugars such sucrose, glucose, fructose, these can be bioprocessed to value added high cost products like antibiotics.

As India produces approximately 10.8 million tonnes of oranges of which $25-30 \%$ turns out to be waste. The reasons for such waste accumulation in the country are due to improper storage, transportation, microbial contamination and pest attack [6]. This huge quantity of rotten orange wastes can be efficiently processed to value added products like penicillin and biogas. In this study, we have attempted to develop a process for the production of Penicillin from orange juice extracted from rotten oranges and methane from orange peel waste. Penicillin was produced using mat culture method (Surface culture) and biomethane was produced by anaerobically digesting orange peel waste with cow dung slurry as inoculum.

\section{Materials and methods}

Rotten and damaged oranges were collected from Palayam vegetable market, Kozhikode, Kerala, India. Pure culture of Penicillium species and fresh cow dung as source of anaerobic bacteria for biomethanation was used for the study. Staphylococcus aureus was used as the test organism for agar-well diffusion assay.

\subsection{Preparation of mat culture for Penicillin production}

Raw/Waste oranges were washed with tap water and its peel was removed. It was squeezed with hand and the juice extract was filtered using a muslin cloth. $250 \mathrm{~mL}$ of juice was taken in $1000 \mathrm{~mL}$ conical flask and sterilized using steam (autoclaved at $15 \mathrm{psi}$ for $15 \mathrm{mins}$ at $121^{\circ} \mathrm{C}$ ). After cooling to room temperature, Penicillium spores were used to inoculate the flask ( 1 X $10^{\mathbf{3}}$ spores $\left./ \mathrm{mL}\right)$ and the flasks were incubated further in room temperature to allow germination of spores and establish a sporulated mat over the liquid media in a time period of 15 days.

\subsection{Supplementation with different nitrogenous} substrates

Different media formulations were made with orange juice extracted from rotten oranges with different additives like ammonium sulphate and peptone. This blending was done in order to supplement nitrogen to optimize the $\mathrm{C}: \mathrm{N}$ ratio, since raw orange juice is a carbon rich media. In addition, raw orange juice extracted with initial $\mathrm{pH}$ of 3.5 , was adjusted to a $\mathrm{pH} 6.5$ with $0.1 \mathrm{~N}$ Calcium hydroxide and resulting media of $\mathrm{pH} 6.5$ was used for growth of Penicillium fungi as mat 


\section{International Journal of Science and Research (IJSR) \\ ISSN (Online): 2319-7064 \\ Index Copernicus Value (2013): 6.14 | Impact Factor (2015): 6.391}

culture.

\section{Bioassay of Penicillin}

After 15 days of growth in mat culture, the flask with sporulated mat was carefully removed from the top of the fermentation broth using a sterile L-shaped glass rod. The remaining fluid was filtered with Whatman no.1 filter paper. The filtrate was tested for its antibiotic activity against Staphylococcus aureus pure culture using agar-well diffusion assay [7]. $100 \mathrm{~mL}$ of agar medium with composition $1 \%$ Glucose, $0.5 \%$ peptone, $0.5 \%$ yeast extract with $\mathrm{pH} 7.5$ was autoclaved for $15 \mathrm{mins}$ at $121^{\circ} \mathrm{C}$ in an autoclave. Sterilized medium was poured to sterile petriplate in laminar airflow chamber $(10 \mathrm{~mL} /$ plate $) . \quad 0.1 \mathrm{~mL}$ of pure culture of Staphylococcus aureus was inoculated into the petriplates and was uniformly spread using sterile glass spreader. A well was cut in centre and $0.5 \mathrm{~mL}$ of the filtrate was poured into the well using a micropipette. The petriplates were incubated at $37^{\circ} \mathrm{C}$ for $24 \mathrm{~h}$. The diameter of the inhibition zone produced was measured in millimeter that represents measure of potency of the antibiotic using each of the formulations with raw orange juice as the basic raw material.

\subsection{Biomethanation of orange peels}

1L conical flasks were taken and marked A and B. In the flask marked A, $700 \mathrm{~mL}$ of fresh cow dung slurry was poured (1 Part fresh cow dung to 1 part water thoroughly mixed using a homogenizer). In the flask marked $\mathrm{B}, 700 \mathrm{~mL}$ of fresh cow dung slurry mixed with $100 \mathrm{~g}$ of orange peels was taken. Both the flasks were closed with one hold rubber stoppers connected with rubber tubes. The fermentation gases produced from the corresponding flasks were collected using three inverted 1L conical flasks completely filled with water and closed with two hold rubber stoppers (water displacement method). When the fermentation gases are conveyed through the single hole connected tubes to the inverted conical flask with water, equal volume of water is displaced, which will be collected in another conical flask and measuring the volume of water displaced gives the quantity of biogas produced in $24 \mathrm{~h}$. The rate of biogas production and quantity depends on the substrate material used.

\section{Results and Discussion}

\subsection{Bioconversion of rotten/waste oranges to Penicillin}

The Fig.1 (a) shows the growth of Penicillium fungus as mat culture over raw orange juice medium. From Fig.1 (a), it is evident that, there is luxuriant growth of Penicillium fungus mat over raw orange juice medium with an initial $\mathrm{pH}$ of 3.5.This proves that orange juice can be used directly as an industrial substrate even without adjustment of $\mathrm{pH}$. Penicillium fungus can be grown as pure culture over a period of 15 days, which will support luxurious mycelial proliferation, as well as intense sporulation that is essential since Penicillin is a secondary metabolite. Fig.1(b) shows the growth of Penicillium fungus as mat culture over raw orange juice medium with $\mathrm{pH}$ adjusted to 6.5.

\subsection{Supplementation of different nitrogenous substrates}

Fig.1 (c,d), gives the picture regarding growth of Penicillium fungi over different nitrogen supplemented media with raw orange juice as the basic substrate. Fig.1 (c) and Fig.1 (d) show sporulation regarding Penicillium fungus in raw orange juice based media, supplemented with peptone and ammonium sulphate respectively.

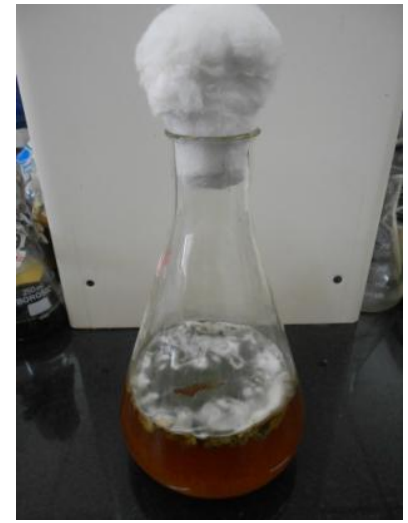

Figure1 (a): Growth of Penicillium fungus in orange juice at pH 3.5.

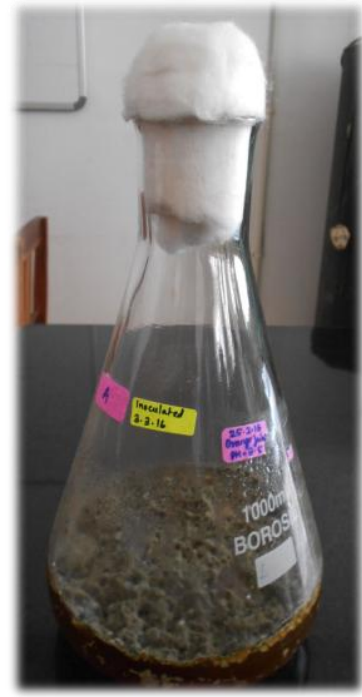

Figure1 (b): Growth of Penicillium fungus in orange juice at $\mathrm{pH} 6.5$.

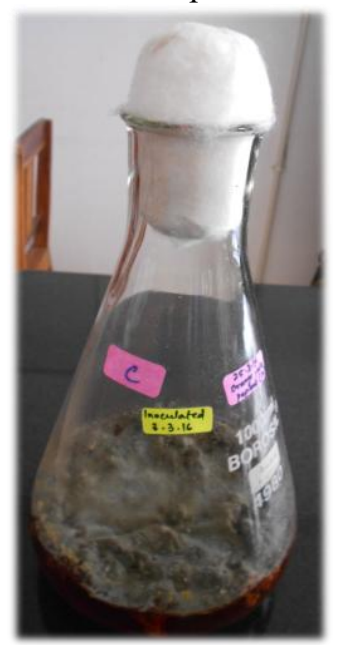

Figure1 (c): Growth of Penicillium fungus in orange juice liquid medium at $\mathrm{pH} 6.5$ with $1 \%$ peptone supplementation. 


\section{International Journal of Science and Research (IJSR) \\ ISSN (Online): 2319-7064}

Index Copernicus Value (2013): 6.14 | Impact Factor (2015): 6.391

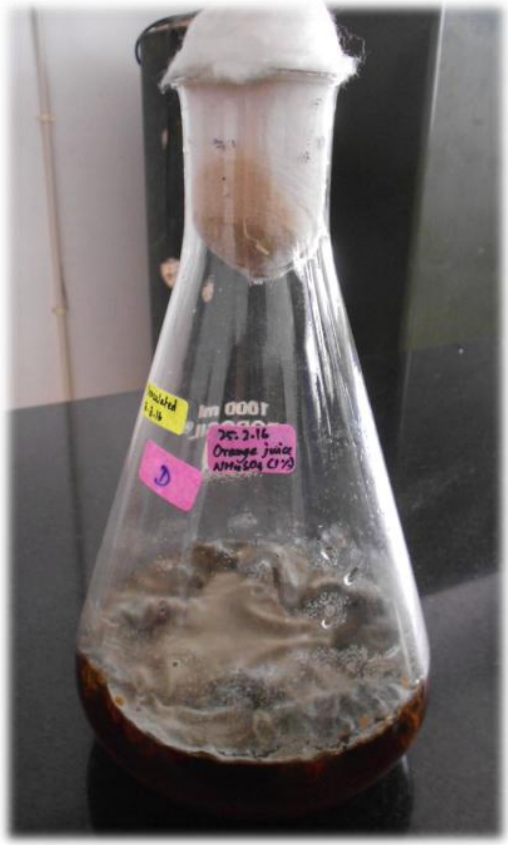

Figure1 (d): Growth of Penicillium fungus in orange juice liquid medium at $\mathrm{pH} 6.5$ with $1 \%$ ammonium sulphate supplementation

\subsection{Agar-well diffusion assay}

Fig.2 (a,b,c,d) shows that agar well diffusion assay data of Penicillin fermentation broth after cell separation from the fermentation broth. The antibiotic activity was given by measuring the diameter of inhibition zone in millimeters._By comparison of the data 2(a), 2(b) and 2(c) and 2(d), it is evident that the potency of the antibiotic produced as per Fig 2(c) using raw orange juice based medium supplemented with peptone gives highest yield of antibiotic. Still, peptone based medium cannot be used for industrial production because the cost of peptone is very high. So, the ammonium sulphate supplemented medium can be used for large scale manufacture of this highly useful and valuable antibiotic, since the cost of ammonium sulphate is very low compared to peptone, considering enormous quantities of wastes available in this country.

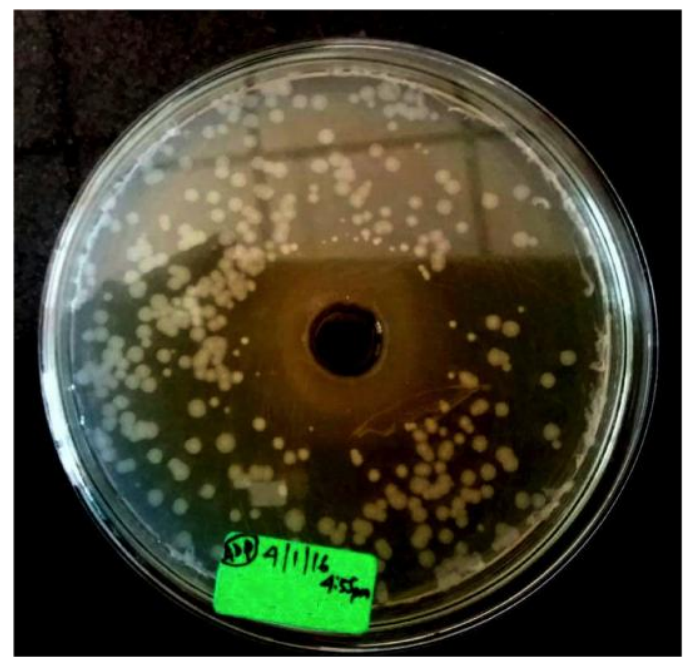

Figure 2 (a): Zone of inhibition (30mm) obtained by the mat culture of Penicillium fungus grown in orange juice based medium at $\mathrm{pH} 3.5$.

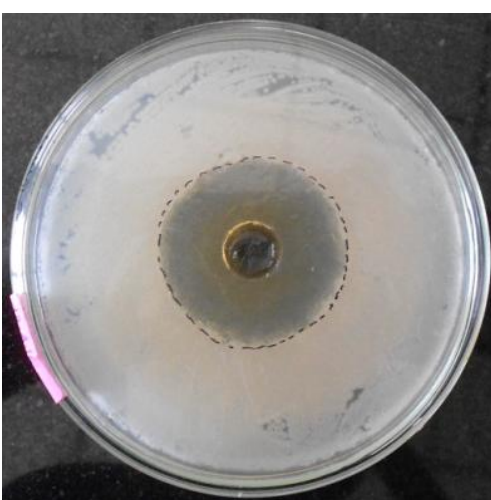

Figure 2 (b): Zone of inhibition (40mm) obtained by the mat culture of Penicillium fungus grown in orange juice based medium at $\mathrm{pH} 6.5$.

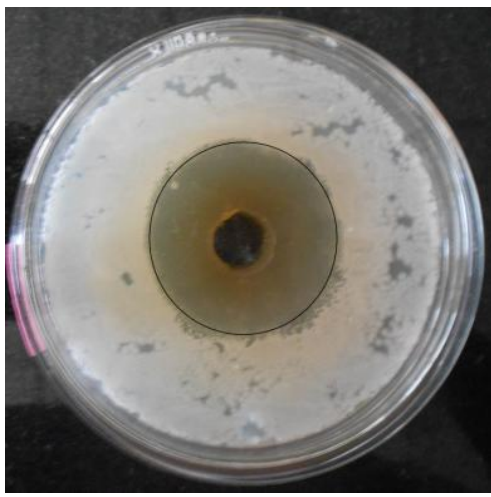

Figure 2 (c): Zone of inhibition (45mm) obtained by the mat culture of Penicillium fungus grown in orange juice based medium with $1 \%$ peptone at $\mathrm{pH} 6.5$.

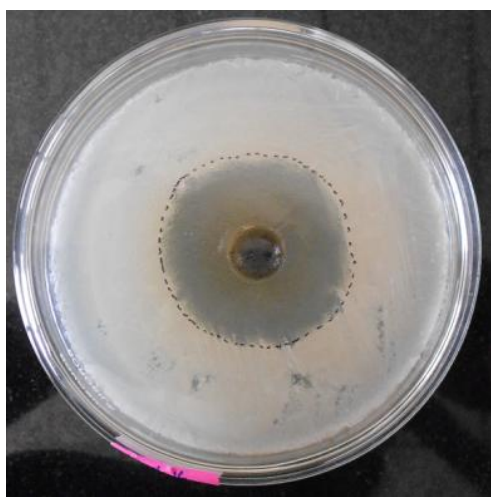

Figure 2 (d): Zone of inhibition ( $41 \mathrm{~mm})$ obtained by the mat culture of Penicillium fungus grown in orange juice based medium with $1 \%$ ammonium sulphate at $\mathrm{pH} 6.5$.

\subsection{Biomethanation of orange peels}

Fig.3 gives the biomethanation data of orange peel waste. Since it contains sugars such as sucrose, glucose, fructose, in addition to pectin, cellulose and hemicellulose, it becomes an attractive substrate for the cost effective biomethanation. In biomethanation process, bacteria convert complex organic materials to biogas through hydrolysis, acidogenesis, acetogenesis and methanogenesis catalyzed by the corresponding microbial consortia. Biogas is an excellent biofuel which usually has $60-65 \%$ methane and $30-35 \% \mathrm{CO}_{2}$ with trace amounts of other gases. Bioconversion of organic wastes to biofuel tackles environmental pollution with bioenergy production, making it a lucrative technology. The 


\section{International Journal of Science and Research (IJSR) \\ ISSN (Online): 2319-7064 \\ Index Copernicus Value (2013): 6.14 | Impact Factor (2015): 6.391}

data shows that bioconversion of orange peel waste produces $68.9 \%$ more biogas compared to control in a time span of 30 days. This data conclusively proves that there is commercial possibility of large scale bioprocessing of huge amounts of orange peel waste which accumulates near fruit processing industries to value added product like biomethane. The technology becomes economically feasible considering the cost spent on waste removal, pollution abatement and revenues incurred as part of imports on crude oil.

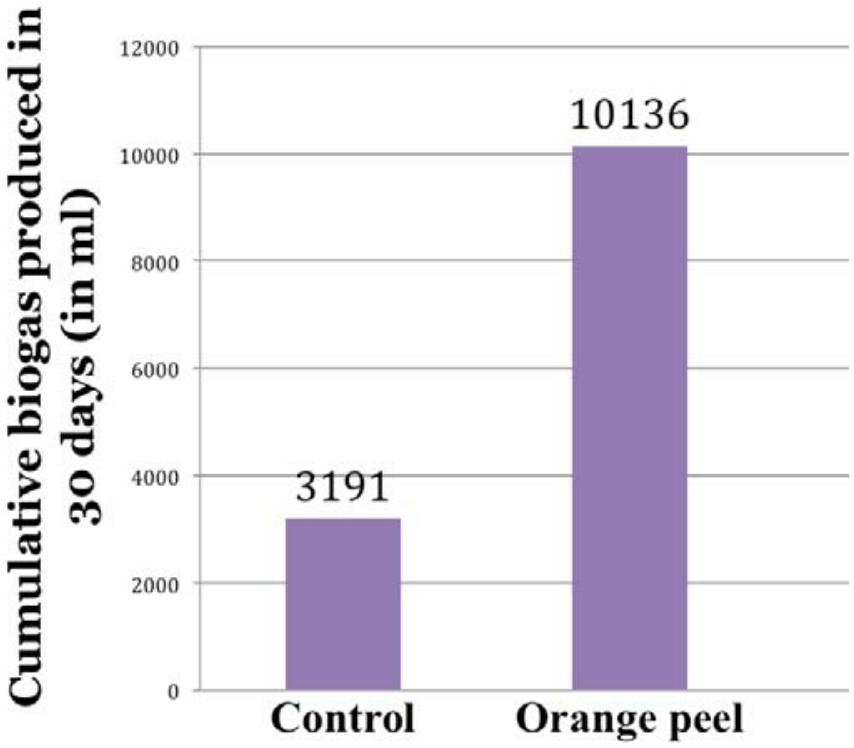

Figure 3: Cumulative biogas production in 30 days

\section{Conclusion}

The rotten/waste orange can efficiently be converted to Penicillin by employing surface mat culture of Penicillium fungus. In addition, biomethanation orange peel wastes is also a technical possibility on an industrial scale.

\section{References}

[1] Rezzadori, K., S. Benedetti, and E. R. Amante. "Proposals for the residues recovery: orange waste as raw material for new products." Food and bioproducts processing 90.4 (2012): 606-614.

[2] Melèndez-Martínez, A.J., Britton, G., Vicario, I.M., Heredia, F.J., 2008. The complex carotenoid pattern of orange juices from concentrate. Food Chem. 109, 546553.

[3] Roussos, P.A., 2011. Phytochemicals and antioxidant capacity of orange (Citrus sinensis (1.) Osbeck cv. Salustiana) juice produced under organic and integrated farming system in Greece. Sci. Hortic. 129, 253-258.

[4] Abecitrus, 2008. História da Laranja e Subprodutos da Laranja. Available from: www.abecitrus.com.br/ (accessed 06.08.08).

[5] Braddock, R.J., 1995. By-products of citrus fruit. Food Technol. 49 (9), 74-77.

[6] "Top Production of Oranges, 2013, United Nations, Food and Agricultural Organization, FAO Statistics. 2013. Retrieved 21 June 2015.
[7] Bennett, John V., et al. "Simplified, accurate method for antibiotic assay of clinical specimens." Applied microbiology 14.2 (1966): 170-177.

\section{Author Profile}

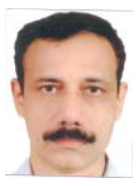

C. Gopinathan is currently working as Associate Professor at Department of Biotechnology, University of Calicut. He received the B.Sc in Botany, M.Sc in Life Sciences with specialization in Microbiology from University of Calicut and M.Tech in Biotechnology from Anna University, Chennai. He was former waste management consultant for Calicut Corporation, Kerala and is, at present consultant for Indian railways. He has handled various bioprocess scaled up projects and published \& presented his research works in leading Biotechnology conferences and International journals including World Biotechnology Congress. His specialization areas include fermentation technology in general and specifically biopesticide production, biogas production from agro waste, low cost mushroom production and high rate aerobic composting. 